\title{
Effectiveness of facetal joint mobilization in T10 syndrome: A case study
}

\author{
Authors \\ Nidhi Vedawala ${ }^{1 *}$,Sakshi Thakrar ${ }^{2}$, Khatri SM $^{3}$ \\ ${ }^{1}$ Tutor, ${ }^{2}$ Assistant Professor, ${ }^{3}$ Principal \\ Nootan College of Physiotherapy, Sankalchand Patel University, Visnagar, Gujarat, India -384 315 \\ *Corresponding Author \\ Nidhi Vedawala
}

Postal Address: Nidhi Vedawala, Fatheh darwaja Road, Near Ghanchiwada, Visnagar - 384315, India

Email: nidhivedawala1591@gmail.com

\section{Abstract}

Currently there is limited evidence about T10 syndrome, its pathophysiology and effectiveness of physiotherapy interventions. We describe the effectiveness of facetal joint mobilization and other passive physiotherapy interventions in a 34 year old female patient. After one week of the treatment there was considerable reduction in her symptoms. Hence we propose that facetal joint mobilization may be considered as an adjunct in the management of T10 syndrome.

Keywords: Lower thoracic pain, T10 syndrome, facetal joint, mobilization, phonophoresis, taping.

\section{Introduction}

Treatment-based classification approach to low back pain describes the model whereby physiotherapist can make treatment decisions based on the patient's clinical presentation. ${ }^{1}$ The primary purpose of the treatment based approach is to identify features at baseline that predict responsiveness to different treatment strategies like manipulation, stabilization or specific exercises. . $^{2,3,4,5}$

However, considering some of the barriers for adopting this classification especially in cases of non-specific low back pain, we felt that customized approach with varied levels of experience could be one of the essential key for favorable outcomes especially for a patient with unusual presentation like T10 syndrome. ${ }^{6}$
Dr Vladimir Janda is credited for describing various muscle imbalance syndromes. Vladimir Janda was a Czech physician and physiotherapist who became known for his work in the field of skeletal muscle, as well as developing systems to assess and correct movement dysfunction. Janda is known as the father of the Czech rehabilitation method, acknowledged as the method of Janda. He described upper crossed syndrome, lower crossed syndrome, layer syndrome etc. Similar to this, the T4 syndrome was described for the first time by Maitland and McGuckin in 1986..$^{7,8,9,10}$ It is described as a collection of symptoms resulting from autonomic dysfunction from the upper thoracic spine.

However, there is limited information about T10 syndrome involving lower thoracic spine related dysfunction producing lower extremity symptoms 
due to possible sympathetic nervous system (SNS) dysfunction and lower thoracic hypomobility. This case report describes a patient with symptoms that gave suspicion of the presence of manual therapy diagnosis in the form of T10 syndrome.

\section{Cases Study}

History: We describe a 34 year old female patient. Her height was 163 centimeters, weight 52 kilograms and body mass index (BMI) was 19.6. She was a married woman with one child. The patient was seen by a physiotherapist and enrolled for daily treatment. She was referred for physiotherapy by a general practioner and diagnosed as a case of non-specific low back. She complained of lower back pain and vague pain and sometimes paresthesis or heaviness in both lower legs since two months. She belonged to a middle socioeconomic class and fair family and social support. She had no history of similar complaint in the past.

Physical examination: Her vital signs were normal. Postural findings included an increased lumbar lordosis. Palpation revealed normal soft tissue and boney contours in the area of symptom. Her New York Posture Rating score indicated no gross abnormality. Local tenderness at tenth and eleventh thoracic vertebrae. Active movements were normal but passive intervertebral movements and passive accessory intervertebral movements were terminally painful with rotations on either side at T10-11 segment.

Procedure: Ethical approval was granted from the Institutional Ethical Committee and the patient gave an informed written consent. Her demographic data, physical examination, pressure pain threshold and visual analogue scale score were recorded.

She was treated with phonophoresis with piroxicam ointment and aquasonic gel with $0.8 \mathrm{~W} / \mathrm{Cm}^{2}$ for 5 minutes with $1 \mathrm{MHz}$ pulsed mode ultrasound with 1:1 pulse ratio, facetal joint mobilization (Figure 1) at T9-10 and T10-11 for three sets of 30 seconds and at the end a crossed dynaplast tape was applied at T10 level with a small cross appearance over T10 spinous process. The treatment protocol was continued for one week and final change in form of outcome measures were recorded before she was instructed to continue the home exercises program.(Figure 2) Before the participation in this study her pain was 5.8 and pressure pain threshold was 7.5 Lbs and after one week of the intervention it was 1.8 and 16.5 Lbs respectively.

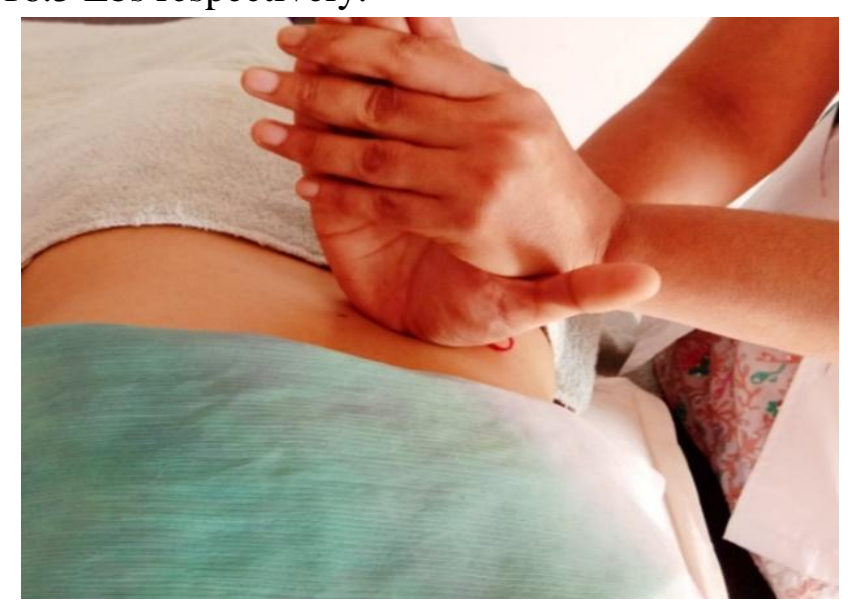

Figure 1: Facetal joint Mobilization technique

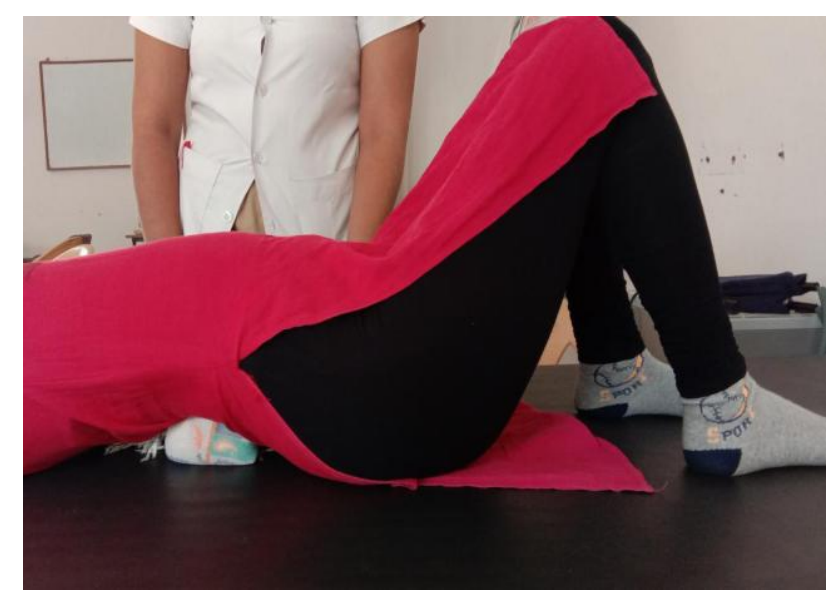

Figure 2: Self Mobilization technique using towel

\section{Discussion}

This case provides information about the presence of the T10 syndrome and its management with physiotherapy interventions. Further, it was noted that significant relief in symptoms were noted in the present case. The term T10 syndrome is uncommon and it appears to be a bunch of symptoms including lower back pain, stiffness, heaviness, paresthesia and tingling in both lower extremities with a possible autonomic dysfunction. ${ }^{11,12,13}$ Facetal joint mobilization may 
have neurophysiological, mechanical, psychological or placebo effect but it appears that mobilization may directly or indirectly work on sympathetic nervous system dysfunction thereby restoring the normal functioning of autonomic nervous system. Passive mobilization may stimulate the sympathetic nervous system and activate the descending pain inhibitory mechanism which increases sympathetic activity and decreases pain due to the release of noradrenaline from the dorsal periaqueductal grey area. ${ }^{14,15}$

\section{Conclusion}

There is lack of studies about the T 10 syndrome but this case study support the idea of possible passive and active physiotherapy interventions like mobilization, phonophoresis and taping as part of overall management.

\section{References}

1. Delitto A, Erhard RE, Bowling RW. A treatment-based classification approach to low back syndrome: identifying and staging patients for conservative treatment. Phys Ther. 1995 Jun; 75(6):470-85; discussion 485-9. (level of evidence $1 \mathrm{~A})$

2. Hebert JJ1, Koppenhaver SL, Walker BF. Subgrouping patients with low back pain: a treatment-based approach to classification. Sports Health. 2011 Nov; 3(6):534-42.

3. Brennan G, Fritz J, Hunter $S$, et al. Identifying subgroups of patients with acute/subacute nonspecific low back pain. Spine. 2006;31:623-631 (level of evidence 1B)

4. Fritz J, Brennan G, Clifford S, et al. [http://journals.lww.com/spinejournal/A bstract/2006/01010/An_Examination_of _the_Reliability_of_a.18.aspx an

examination of the reliability of a classification algorithm for subgrouping patients with low back pain]. Spine. 2006; 31:77-82. (Level of Evidence 1B)
5. Fritz J, Delitto A, Erhard R. Comparison of classification-based physical therapy with therapy based on clinical practice guidelines for patients with acute low back pain. Spine. 2003; 28:1363-1372. (Level of evidence 1B)

6. Liba Sheeran Philippa Coales Valerie Sparkes: Clinical challenges of classification based targeted therapies for non-specific low back pain: What do physiotherapy practitioners and managers think? Manual Therapy. Volume 20, Issue 3, June 2015, Pages 456-462

7. Phillip Page, Clare Frank, Robert Lardner: Assessment and Treatment of Muscle Imbalance: The Janda Approach. Human Kinetics. 2010

8. Liebenson, Craig. "In Memory of Professor Vladimir Janda (1928-2002)". https://www.dynamicchiropractic.com/m pacms/dc/article.php?id=9001 retrived on 2018-11-07

9. Maitland, G.D., Manipulation der Wirbelsäule 1986, Berlin: Springer.

10. McGuckin, N., The T4 Syndrome, in Modern Manual Therapy of the vertebral column, G.P. Grieve, Editor 1986, Churchill Livingstone: Edinburgh. p. $370-376$.

11. Muhammad Nazim Farooq: Lower thoracic syndrome Pak J Med Sci 2017 Vol. 33 No. 3 767-769.

12. Geerse WK: Bilateral leg symptoms--the T10 syndrome? Man Ther. 2012 Jun; 17(3):251-4. Epub 2011 Sep 22. doi: 10.1016/j.math.2011.08.003.

13. https://www.researchgate.net/publication/5 1670630_Bilateral_leg_symptoms__The_T10_syndrome [accessed Nov 18 2018].

14. Perry J, Green A. An investigation into the effects of a unilaterally applied lumbar mobilization technique on peripheral sympathetic nervous system activity in the 
lower limbs. Man Ther. 2008; 13(6):492499. doi:10.1016/j. math.2007.05.015

15. Mark D Bishop, ${ }^{* 1,2,3}$ Rafael TorresCueco, ${ }^{4}$ Charles W Gay, ${ }^{1,2}$ Enrique LluchGirbés, ${ }^{4}$ Jason M Beneciuk, ${ }^{1,5}$ and Joel E Bialosky:What effect can manual therapy have on a patient's pain experience?Pain Manag. 2015 Nov; 5(6): 455464.doi: [10.2217/pmt.15.39]. 